\title{
EXPONENTIAL BEHAVIOR IN THE PRESENCE OF DEPENDENCE IN RISK THEORY
}

\author{
HANSJÖRG ALBRECHER, ${ }^{*}$ Austrian Academy of Sciences, Linz, \\ and Graz University of Technology \\ JEF L. TEUGELS, ${ }^{* *}$ Katholieke Universiteit Leuven and EURANDOM
}

\begin{abstract}
We consider an insurance portfolio situation in which there is possible dependence between the waiting time for a claim and its actual size. By employing the underlying random walk structure we obtain explicit exponential estimates for infinite- and finitetime ruin probabilities in the case of light-tailed claim sizes. The results are illustrated in several examples, worked out for specific dependence structures.
\end{abstract}

Keywords: Dependence; risk model; copula; renewal theory; Wiener-Hopf theory

2000 Mathematics Subject Classification: Primary 62P05

Secondary 60G50; 60K05

\section{Introduction}

The classical risk theory describing characteristics of the surplus process of a portfolio of insurance policies usually relies on the assumption of independence of claim sizes and claim interoccurrence times. However, in many applications this assumption is too restrictive and generalizations to dependent scenarios are called for. In recent years, a number of results on ruin probabilities have been obtained for models that allow for specific types of dependence (see [2] for a survey on the subject).

One traditional technique of deriving results in risk theory is to describe the surplus process as a random walk with independent increments between two claim instances. It is well known that if the Laplace transform of the distribution of the increments exists in a left neighborhood of the origin, then the asymptotic behavior of ruin probabilities in infinite and finite time are determined by properties of the Laplace transform in that region.

In this paper we take up this random walk approach. However, we allow the interclaim time and its subsequent claim size to be dependent according to an arbitrary copula structure, thus separating the dependence behavior from the properties of the marginal distributions. The introduction of dependence modifies the shape of the Laplace transform, but the random walk structure is preserved and we can derive asymptotic results for the ruin function by studying properties of this Laplace transform. This approach seems to be new; the present paper is not meant to be an exhaustive treatment of the subject - it should rather be seen as a starting point.

In Section 2 we present some preliminaries on random walk techniques and their connection with ruin theory. In Section 3 the Laplace transform of the increment distribution of the random walk is introduced and some of its properties are discussed. In Section 4 we rederive the

Received 15 March 2004; revision received 29 November 2005.

* Postal address: Department of Mathematics, Graz University of Technology, Steyrergasse 30, Graz, 8010, Austria. Email address: albrecher@tugraz.at

** Postal address: Department of Mathematics, Katholieke Universiteit Leuven, de Croylaan 54, Heverlee, 3001, Belgium. 
Cramér-Lundberg approximation for the infinite-time ruin probability in terms of random walk quantities, and discuss the behavior of the adjustment coefficient in the presence of dependence. Explicit exponential estimates of finite-time ruin probabilities for these dependent scenarios are given in Sections 5 and 6, and discussed in some detail for several specific dependence structures. We conclude in Section 7.

\section{Preliminaries}

We start by introducing the main quantities from both ruin and random walk theory.

\subsection{Portfolio quantities}

Let claim sizes arrive according to a renewal process with interclaim times $\left\{T_{i}, i=1,2, \ldots\right\}$ and $T_{0}=0$. The generic interclaim time $T$ has distribution $F_{T}$. Let the claim sizes $\left\{U_{i}, i=\right.$ $1,2, \ldots\}$ form another renewal process, generated by the random variable $U$ with distribution $F_{U}$. We assume that there is constant payment of premiums at a rate $c$. We will normally assume that the bivariate process $\left\{\left(U_{i}, T_{i}\right), i=1,2, \ldots\right\}$ is a bivariate renewal process generated by the pair $(U, T)$. In this case the quantities $\left\{X_{i}:=U_{i}-c T_{i}, i \geq 1\right\}$ are independent and identically distributed, which is necessary in the random walk approach that we are following. The random variable $X=U-c T$ will be called the generic variable. As a special case we mention the famous Sparre Andersen model, in which the two processes are independent.

Denote by $R_{n}$ the risk reserve immediately after payment of the $n$th claim. Then $R_{0}=u$ is obviously the initial reserve, while, for $n \geq 0, R_{n+1}=R_{n}+c T_{n+1}-U_{n+1}$.

\subsection{Random walks}

To introduce a random walk, for all $n \geq 1$ define $X_{n}=U_{n}-c T_{n}$, which can be interpreted as the loss between the $(n-1)$ th and the $n$th claims. Then, with $S_{0}=0$ and $S_{n+1}=S_{n}+X_{n+1}$, $n \geq 0$, we can express $R_{n}=u-S_{n}$ in terms of the random walk $\left\{S_{n}\right\}$. Let

$$
K(x)=\mathrm{P}\{X \leq x\}=\mathrm{P}\{U-c T \leq x\}
$$

be the distribution of the generic variable $X$. Without further ado we assume that

$$
\mathrm{E}\{X\}=\mathrm{E}\{U\}-c \mathrm{E}\{T\}<0
$$

as, otherwise, the insurance company will be ruined with probability 1 .

We use the terminology and notation from general random walk theory (see, e.g. [11]). At every instant, the random walk $\left\{S_{n}, n \geq 0\right\}$ itself is determined by the $n$-fold convolution

$$
K^{* n}(x)=\mathrm{P}\left\{S_{n} \leq x\right\},
$$

where $K^{* 0}(x)$ is the unit-step distribution at the origin. The characteristic function of $X$ will be denoted by

$$
\kappa(\zeta):=\mathrm{E}\{\exp (\mathrm{i} \zeta X)\}, \quad \zeta \in \mathbb{R} .
$$

The following quantities are among the prime objects of study in random walk theory. The first upgoing ladder index is defined by $N:=\inf \left\{n>0: S_{n}>0\right\}$ and the corresponding first upgoing ladder height is then $S_{N}$. A famous result by Baxter (see [11, p. 204]) gives the hybrid transform of the pair $\left(N, S_{N}\right)$. For $|s|<1$ and $\theta \geq 0$,

$$
\mathrm{E}\left\{s^{N} \mathrm{e}^{-\theta S_{N}}\right\}=1-\exp \left(-\sum_{n=1}^{\infty} \frac{s^{n}}{n} \int_{0+}^{\infty} \mathrm{e}^{-\theta x} \mathrm{~d} K^{* n}(x)\right) .
$$


In the notation of Feller [5, p. 540], the above hybrid transform appears as the right Wiener-Hopf factor of the characteristic function $\kappa(\cdot)$. By this we mean that

$$
1-s \kappa(\zeta)=(1-\chi(s, \zeta))(1-\tilde{\chi}(s, \zeta)),
$$

where $\chi(s, \zeta):=\mathrm{E}\left\{s^{N} \mathrm{e}^{\mathrm{i} \zeta S_{N}}\right\}$ and $\tilde{\chi}(\cdot, \cdot)$ refers to the analogous quantity for the (weak) downgoing ladder index and ladder height.

The maxima of the random walk are defined by $M_{0}=0$ and, for $n \geq 1$,

$$
M_{n}=\max \left(0, S_{1}, \ldots, S_{n}\right) .
$$

We denote the distribution of $M_{n}$ by $G_{n}(x):=\mathrm{P}\left\{M_{n} \leq x\right\}$. The supremum of the random walk is defined by $M_{\infty}=\sup \left(0, S_{1}, S_{2}, \ldots\right)$, and, furthermore, $G(x):=\mathrm{P}\left\{M_{\infty} \leq x\right\}$. A classification quantity that often appears is given by

$$
B(s):=\sum_{n=1}^{\infty} \frac{s^{n}}{n} \mathrm{P}\left\{S_{n}>0\right\} .
$$

Now, $B:=B(1)<\infty$ if and only if $M_{\infty}<\infty$ almost surely; moreover, then $\lim \sup S_{n}=$ $-\infty$. In particular, since $\mathrm{E}\{X\}<0$, it automatically follows that $B<\infty$.

For further reference, it is necessary to include information on the distributions $\left\{G_{n}(\cdot)\right\}$. We introduce the generating function for this sequence. Let $|s|<1$ and define

$$
G(s, x):=\sum_{n=0}^{\infty} G_{n}(x) s^{n} .
$$

It follows from the Spitzer-Baxter identity [5, p. 569], [11, p. 220] that

$$
\int_{0}^{\infty} \mathrm{e}^{\mathrm{i} \zeta x} G(s, \mathrm{~d} x)=\frac{\mathrm{e}^{-B(s)}}{(1-s)(1-\chi(s, \zeta))}
$$

Part of the Wiener-Hopf factor in (2.2) appears in the expression for the Laplace transform of the supremum. Indeed, it follows from (2.3) that, at least for $\theta \geq 0$,

$$
\int_{0}^{\infty} \mathrm{e}^{-\theta x} \mathrm{~d} G(x)=\exp \left(-\sum_{n=1}^{\infty} \frac{1}{n} \int_{0}^{\infty}\left(1-\mathrm{e}^{-\theta x}\right) \mathrm{d} K^{* n}(x)\right)=\frac{\mathrm{e}^{-B}}{1-\mathrm{E}\left\{\mathrm{e}^{-\theta S_{N}}\right\}} .
$$

\subsection{Connections}

The links between the random walk concepts and the risk quantities are straightforward. Let us define the time of ruin with initial reserve $u$ as

$$
\tau(u):=\inf \left\{n: u<S_{n}\right\} .
$$

Then ruin will occur at the $n$th claim if the total loss, expressed in terms of the random walk $S_{n}$, has annihilated the initial surplus. In terms of the maximum, we obtain the fundamental relation

$$
\{\tau(u)>n\}=\left\{M_{n} \leq u\right\} .
$$

This equation immediately implies that ruin will occur in finite time, but after the $n$th claim if and only if $M_{n}$ does not overshoot $u$ but $M_{\infty}$ will. Hence,

$$
\mathrm{P}\{n<\tau(u)<\infty\}=\mathrm{P}\left\{M_{n} \leq u<M_{\infty}\right\}=G_{n}(u)-G(u) .
$$




\section{The generic variable}

While it is not fully necessary to do so, we will assume from now on that the joint distribution function $F_{U, T}(u, t)=\mathrm{P}\{U \leq u, T \leq t\}$ has a bivariate density, $f_{U, T}$. We are interested in the distribution, $K$, of the generic variable $X=U-c T$. Recall that $\mathrm{E}\{X\}=\mathrm{E}\{U\}-c \mathrm{E}\{T\}<0$. Obviously, the density of $X$ exists and is given by

$$
k(z)=\frac{1}{c} \int_{0}^{\infty} f_{U, T}\left(u, \frac{u-z}{c}\right) \mathrm{d} u .
$$

The characteristic function, $\kappa(\zeta)$, of $K$ can be obtained from the joint characteristic function

$$
\mathrm{E}\left\{\mathrm{e}^{\mathrm{i} \zeta_{1} U+\mathrm{i} \zeta_{2} T}\right\}=\int_{0}^{\infty} \mathrm{d} u \int_{0}^{\infty} \mathrm{d} t \mathrm{e}^{\mathrm{i} \zeta_{1} u+\mathrm{i} \zeta_{2} t} f_{U, T}(u, t)
$$

by choosing $\zeta_{1}=\zeta$ and $\zeta_{2}=-c \zeta$. In general, we cannot be sure that $\kappa(\zeta)$ exists for any nonreal value of $\zeta$.

\subsection{The double Laplace transform}

As shown by Widder [16, p. 39], the distribution $K$ will have an exponentially bounded right tail if and only if the double Laplace transform $\hat{K}(\theta):=\kappa(\mathrm{i} \theta)$ converges in a left neighborhood of the origin. We will therefore replace $\zeta$ by i $\theta$, to obtain the (two-sided) Laplace transform of $X$ rather than the characteristic function. Alternatively, the left abscissa of convergence, $-\sigma_{K}$, of $\hat{K}(\theta)$ should be strictly negative (in which case $K$ is said to be super-exponential). We will generally write $-\sigma_{Y} \equiv-\sigma_{H}$ for the left abscissa of convergence of the Laplace transform of a random variable $Y$ with distribution $H$.

The main object of study is thus

$$
\hat{K}(\theta)=\int_{u=0}^{\infty} \int_{t=0}^{\infty} \mathrm{e}^{-\theta(u-c t)} f_{U, T}(u, t) \mathrm{d} u \mathrm{~d} t,
$$

and we will restrict our analysis in this paper to cases in which $\sigma_{X}>0$. Notice that the balance condition tells us that $\hat{K}^{\prime}(0)=-\mathrm{E}\{X\}>0$. Also note that, since $T \geq 0$, we have $X \leq U$. Hence, we always have $\sigma_{X} \geq \sigma_{U} \geq 0$, meaning that exponentially bounded claim sizes automatically lead to an exponentially bounded generic variable.

It is well known that every joint distribution function can be expressed as a copula function of its marginal distributions (this copula representation being unique for continuous multivariate distribution functions); thus, $F_{U, T}(u, t)=C\left(F_{U}(u), F_{T}(t)\right)$ for some copula $C$. This approach allows us to completely separate the dependence structure from the properties of the univariate marginals (for a survey on copulas we refer the reader to Joe [8] or Nelsen [10]). In what follows we try to formulate our results in terms of copulas. Using the identity

$$
1-F_{U, T}(x, \infty)-F_{U, T}(\infty, y)+F_{U, T}(x, y)=\int_{x}^{\infty} \mathrm{d} u \int_{y}^{\infty} \mathrm{d} t f_{U, T}(u, t),
$$

for every $\theta>-\sigma_{X}$ we obtain

$$
\begin{aligned}
\hat{K}(\theta) & -\hat{F}_{U}(\theta)-\hat{F}_{T}(-c \theta)+1 \\
& =-c \theta^{2} \int_{0}^{1} \mathrm{e}^{-\theta F_{U}^{-1}(a)} \int_{0}^{1} \mathrm{e}^{c \theta F_{T}^{-1}(b)}(1-a-b+C(a, b)) \mathrm{d} F_{T}^{-1}(b) \mathrm{d} F_{U}^{-1}(a)
\end{aligned}
$$


or, equivalently,

$$
\begin{aligned}
\hat{K}(\theta)= & \hat{F}_{U}(\theta) \hat{F}_{T}(-c \theta) \\
& -c \theta^{2} \int_{0}^{1} \mathrm{e}^{-\theta F_{U}^{-1}(a)} \int_{0}^{1} \mathrm{e}^{c \theta F_{T}^{-1}(b)}(C(a, b)-a b) \mathrm{d} F_{T}^{-1}(b) \mathrm{d} F_{U}^{-1}(a) .
\end{aligned}
$$

The above formula explicitly shows how $\hat{K}(\theta)$ depends on the copula and the marginals. If the copula function is absolutely continuous, we can also write (3.1) as

$$
\hat{K}(\theta)=\int_{u=0}^{\infty} \int_{t=0}^{\infty} \mathrm{e}^{-\theta(u-c t)} f_{U}(u) f_{T}(t) c\left(F_{U}(u), F_{T}(t)\right) \mathrm{d} t \mathrm{~d} u,
$$

where $c(a, b)=\partial^{2} C(a, b) / \partial a \partial b$.

We will now briefly discuss three simple copulas that can be viewed as extremal cases of dependence.

Example 3.1. (The independence copula.) The independence copula is given by $C_{\mathrm{I}}(a, b):=$ $a b$, and we will denote the corresponding distribution by $K_{\mathrm{I}}$. If $U$ and $T$ are independent then $\hat{K}_{\mathrm{I}}(\theta)=\hat{F}_{U}(\theta) \hat{F}_{T}(-c \theta)$. Clearly $\hat{K}_{\mathrm{I}}(\theta)$ then exists for all $\theta \in\left(-\sigma_{U},(1 / c) \sigma_{T}\right)$. Hence, $\sigma_{K_{\mathrm{I}}}=\sigma_{U}$.

Note that, for an arbitrary copula, we have $\hat{K}^{\prime}(0)=-\mathrm{E}\{U-c T\}=\hat{K}_{\mathrm{I}}^{\prime}(0)$, since this is a property of the marginal distributions $U$ and $T$ only. However, the difference of the second derivatives $\hat{K}^{\prime \prime}(0)$ and $\hat{K}_{\mathrm{I}}^{\prime \prime}(0)$ already reflects the dependence structure through the covariance of $U$ and $T\left(\hat{K}^{\prime \prime}(0)<\hat{K}_{\mathrm{I}}^{\prime \prime}(0)\right.$ for $\operatorname{cov}(U, T)>0$, and conversely).

Example 3.2. (The comonotone copula.) The strongest possible positive dependence between $U$ and $T$ is attained for the comonotone copula, $C_{\mathrm{M}}(a, b):=\min (a, b)$, corresponding to the distribution $K_{\mathrm{M}}$. This copula is singular and its Laplace transform is given by

$$
\hat{K}_{\mathrm{M}}(\theta)=\int_{0}^{\infty} \exp \left(-\theta\left(u-c F_{T}^{-1}\left(F_{U}(u)\right)\right)\right) f_{U}(u) \mathrm{d} u .
$$

In the special case in which $U$ and $T$ are exponential marginal distributions (with parameters $\lambda_{1}$ and $\lambda_{2}$, respectively), we obtain

$$
\hat{K}_{\mathrm{M}}(\theta)=\frac{\lambda_{1}}{\lambda_{1}+\theta\left(1-c \lambda_{1} / \lambda_{2}\right)} .
$$

For the comonotone (and some related) copulas, we can construct examples of heavy-tailed distributions $F_{U}$ that still lead to $\sigma_{K}>0$.

Example 3.3. (The countermonotone copula.) The strongest possible negative dependence between $U$ and $T$ is attained for the (singular) countermonotone copula,

$$
C_{\mathrm{W}}(a, b)=\max (a+b-1,0),
$$

corresponding to the distribution $K_{\mathrm{W}}$. The associated Laplace transform can be derived to be

$$
\begin{aligned}
\hat{K}_{\mathrm{W}}(\theta) & =\int_{0}^{\infty} \exp \left(-\theta\left(u-c F_{T}^{-1}\left(1-F_{U}(u)\right)\right)\right) f_{U}(u) \mathrm{d} u \\
& =\int_{0}^{1} \exp \left(-\theta\left(F_{U}^{-1}(v)-c F_{T}^{-1}(1-v)\right)\right) \mathrm{d} v .
\end{aligned}
$$


Trivially, $F_{U}^{-1}(v)-c F_{T}^{-1}(1-v) \leq F_{U}^{-1}(v)$; thus, for $\theta \leq 0$,

$$
\hat{K}_{\mathrm{W}}(\theta) \leq \int_{0}^{1} \mathrm{e}^{-\theta F_{U}^{-1}(v)} \mathrm{d} v=\hat{F}_{U}(\theta),
$$

implying that $\sigma_{K_{\mathrm{W}}} \geq \sigma_{U}$.

In the special case in which $U$ and $T$ are exponential marginal distributions (again with respective parameters $\lambda_{1}$ and $\lambda_{2}$ ), we obtain

$$
\hat{K}_{\mathrm{W}}(\theta)=B\left(1+\frac{\theta}{\lambda_{1}}, 1-\frac{c \theta}{\lambda_{2}}\right), \quad-\lambda_{1}<\theta<c \lambda_{2},
$$

in terms of a beta function.

Note that the above comonotone and countermonotone copulas are those degenerate cases of bivariate dependence in which one random variable is a deterministic monotone function of the other.

Remark 3.1. Since any copula $C(a, b)$ is itself a joint distribution function with uniform marginals, we have $C_{\mathrm{W}}(a, b) \leq C(a, b) \leq C_{\mathrm{M}}(a, b)$ for all $a$ and $b, 0 \leq a, b \leq 1$. (These inequalities are often referred to as the Fréchet-Hoeffding bounds.) By virtue of (3.2), we thus find that, for fixed marginals, the Laplace transform $\hat{K}(\theta)$ is bounded by $\hat{K}_{\mathrm{M}}(\theta) \leq \hat{K}(\theta) \leq$ $\hat{K}_{\mathrm{W}}(\theta)$ for those values of $\theta$ at which the quantities are defined.

\section{Infinite-time ruin}

Owing to the connection between ruin and the random walk, $\mathrm{P}\{\tau(u)<\infty\}=1-G(u)$, where $G(u)=\mathrm{P}\left\{M_{\infty} \leq u\right\}$ is given by (2.3). From the Wiener-Hopf factorization (2.2) at $s=1$, we know that $1-\hat{K}(\theta)=\left(1-\mathrm{E}\left\{\mathrm{e}^{-\theta S_{N}}\right\}\right)(1-\tilde{\chi}(1, \mathrm{i} \theta))$. However, then the abscissa of convergence of $\hat{K}(\theta)$ is the same as that of $\mathrm{E}\left\{\mathrm{e}^{-\theta S_{N}}\right\}$ and, therefore, also that of $G$. Hence, $\sigma_{K}=\sigma_{G}$.

Now assume that there exists an adjustment coefficient, $R>0$, for which $\mathrm{E}\left\{\mathrm{e}^{R S_{N}}\right\}=1$. The Wiener-Hopf factorization above then implies that $\hat{K}(-R)=1$. With $\beta:=\theta+R$ in (2.4), we obtain

where

$$
\int_{0}^{\infty} \mathrm{e}^{-\beta x} \mathrm{~d}\left(\int_{0}^{x} \mathrm{e}^{R y} \mathrm{~d} G(y)\right)=\frac{\mathrm{e}^{-B}}{1-\mathrm{E}\left\{\mathrm{e}^{-\beta \tilde{S}_{N}}\right\}},
$$

$$
\mathrm{P}\left\{\tilde{S}_{N} \leq x\right\}:=\int_{0}^{x} \mathrm{e}^{R y} \mathrm{dP}\left\{S_{N} \leq y\right\} .
$$

It is clear that the function $H_{1}(x):=\int_{0}^{x} \mathrm{e}^{R y} \mathrm{~d} G(y)$ is then a renewal function. By Blackwell's renewal theorem (see [4]) we have

$$
H_{1}(x+y)-H_{1}(x) \stackrel{\mathrm{D}}{\rightarrow} \frac{\mathrm{e}^{-B}}{\mathrm{E}\left\{\tilde{S}_{N}\right\}} y=: c_{1} y, \quad x \rightarrow \infty,
$$

where $\stackrel{\text { D }}{\rightarrow}$ ' denotes convergence in distribution. However, since $\mathrm{d} G(x)=\mathrm{e}^{-R x} \mathrm{~d} H_{1}(x)$, we have

$$
\begin{aligned}
\mathrm{e}^{R u}(1-G(u)) & =\mathrm{e}^{R u} \int_{u}^{\infty} \mathrm{e}^{-R x} \mathrm{~d} H_{1}(x)=\int_{0}^{\infty} \mathrm{e}^{-R w} H_{1}(u+\mathrm{d} w) \\
& \rightarrow c_{1} \int_{0}^{\infty} \mathrm{e}^{-R w} \mathrm{~d} w=\frac{c_{1}}{R}
\end{aligned}
$$


If we return to the original quantities, we find that, as $u \rightarrow \infty$,

$$
\mathrm{P}\{\tau(u)<\infty\} \sim \frac{\mathrm{e}^{-B}}{R \mathrm{E}\left\{S_{N} \mathrm{e}^{R S_{N}}\right\}} \mathrm{e}^{-R u},
$$

which completes a particularly transparent proof, in the spirit of Feller [5], of the well-known Cramér-Lundberg approximation for the infinite-time ruin probability. It has been derived in various other ways in the literature (see, e.g. [12]). In the above version, the constant in the approximation is expressed as a function of quantities related to the underlying random walk.

Remark 4.1. Note that the classical form of the Cramér-Lundberg approximation for the compound Poisson model, where $U$ and $T \stackrel{\mathrm{D}}{=} \operatorname{Exp}(1 / \lambda)$ are independent, can be obtained from (4.2) by using the corresponding Wiener-Hopf factorization,

$$
1-\frac{\lambda / c}{\lambda / c-\mathrm{i} \zeta} \mathrm{E}\left\{\mathrm{e}^{\mathrm{i} \zeta U}\right\}=\left(1-\frac{\lambda / c}{\lambda / c-\mathrm{i} \zeta}\right)\left(1-\frac{\lambda}{c} \frac{1-\mathrm{E}\left\{\mathrm{e}^{\mathrm{i} \zeta U}\right\}}{\mathrm{i} \zeta}\right),
$$

from which it follows that

$$
\mathrm{E}\left\{\mathrm{e}^{-\theta S_{N}}\right\}=1+\frac{\lambda}{c \theta}\left(1-\mathrm{E}\left\{\mathrm{e}^{-\theta U}\right\}\right)
$$

and, thus,

$$
\mathrm{E}\left\{S_{N} \mathrm{e}^{R S_{N}}\right\}=\frac{\lambda \mathrm{E}\left\{U \mathrm{e}^{R U}\right\}-c}{c R} .
$$

This together with $\mathrm{e}^{-B}=\mathrm{P}\{N=\infty\}=1-\lambda \mathrm{E}\{U\} / c$ leads to the well-known expression

$$
\mathrm{P}\{\tau(u)<\infty\} \sim \frac{c-\lambda \mathrm{E}\{U\}}{\lambda \mathrm{E}\left\{U \mathrm{e}^{R U}\right\}-c} \mathrm{e}^{-R u}, \quad u \rightarrow \infty .
$$

In the general case, it follows from (4.2) that the asymptotic behavior of the ruin probability is determined by the value of the adjustment coefficient, $R$, defined by $\hat{K}(-R)=1$. Let us fix the marginal distributions of $U$ and $T$ and define $R_{\mathrm{I}}$ to be the adjustment coefficient in the case in which $U$ and $T$ are independent, i.e. $\hat{F}_{U}\left(-R_{\mathrm{I}}\right) \hat{F}_{T}\left(c R_{\mathrm{I}}\right)=1$. If $U$ and $T$ are also positively quadrant dependent (that is, $\mathrm{P}\{U>u, T>t\} \geq \mathrm{P}\{U>u\} \mathrm{P}\{T>t\}$ for all $u$ and $t$ with $0 \leq u, t<\infty)$, then we have $C(a, b) \geq a b$, for all $a$ and $b, 0 \leq a, b \leq 1$, for its copula. It thus follows from (3.3) that

$$
\hat{K}(\theta) \leq \hat{K}_{\mathrm{I}}(\theta) \text { for all } \theta \in\left(-\sigma_{K}, 0\right),
$$

whence $R>R_{\mathrm{I}}$. Conversely, for negatively quadrant-dependent variables $U$ and $T$ we find that

$$
\hat{K}(\theta) \geq \hat{K}_{\mathrm{I}}(\theta) \text { for all } \theta \in\left(-\sigma_{K}, 0\right),
$$

implying that $R<R_{\mathrm{I}}$. In order to quantify the difference between $R$ and $R_{\mathrm{I}}$ we can use the Lagrange expansion, by which the value of $R$ can be expressed in terms of properties of the Laplace transform at the value of the adjustment coefficient of the independence case. In this way we obtain

$$
\begin{aligned}
-R & =-R_{\mathrm{I}}+\left.\sum_{n=1}^{\infty} \frac{\mathrm{d}^{n-1}}{\mathrm{~d} w^{n-1}}\left(\frac{w+R_{\mathrm{I}}}{\hat{K}(w)-\hat{K}\left(-R_{\mathrm{I}}\right)}\right)^{n}\right|_{w=-R_{\mathrm{I}}} \frac{\left(1-\hat{K}\left(-R_{\mathrm{I}}\right)\right)^{n}}{n !} \\
& =-R_{\mathrm{I}}+\frac{1-\hat{K}\left(-R_{\mathrm{I}}\right)}{\hat{K}^{\prime}\left(-R_{\mathrm{I}}\right)}-\frac{1}{2} \frac{\hat{K}^{\prime \prime}\left(-R_{\mathrm{I}}\right)}{\hat{K}^{\prime}\left(-R_{\mathrm{I}}\right)^{3}}\left(1-\hat{K}\left(-R_{\mathrm{I}}\right)\right)^{2}+\cdots,
\end{aligned}
$$


the series being convergent as long as both the inverse of $\hat{K}(\theta)$ is analytic in the domain under consideration and $\hat{K}^{\prime}\left(-R_{\mathrm{I}}\right) \neq 0$. This formula is particularly useful for investigating the sensitivity of the adjustment coefficient to the presence of dependency between $U$ and $T$. Some specific examples in which $R$ can even be expressed explicitly as a function of a dependence measure will be given in the next section.

Remark 4.2. Although quadrant dependence is one of the weakest dependence concepts, owing to (3.3) it turns out to be sufficient for deriving inequalities for the adjustment coefficient. Other dependence concepts, such as association, tail monotonicity, stochastic monotonicity, and likelihood ratio dependence, imply quadrant dependence, and (4.3) and (4.4) thus follow accordingly for these concepts.

In general, whenever there is a concordance ordering, $C_{1} \succ C_{2}$, between copulas $C_{1}$ and $C_{2}$ (i.e. $C_{1}(a, b) \geq C_{2}(a, b)$ for all $a$ and $b, 0 \leq a, b \leq 1$ ), by (3.3) we have $R_{1} \geq R_{2}$. (For a survey of dependence concepts and orderings we refer the reader to Joe [8] or Nelsen [10].)

\section{Finite-time ruin}

We now adapt a result from the literature on random walk theory [13], [14]. The exponential speed of convergence of a random walk towards its upper limit immediately translates into the following finite-time ruin estimate for our risk process.

Theorem 5.1. Assume that

(i) $-\infty \leq \mathrm{E}\{X\}<0$;

(ii) $\hat{K}(\theta)$ converges for $-\sigma_{K}<\theta \leq 0$, where $\sigma_{K}>0$;

(iii) for some $\omega \in\left(0, \sigma_{K}\right), \hat{K}(\theta)$ attains a minimum, $\hat{K}(-\omega)=: \gamma<1$ (see Figure 1).

Then, for all finite $u \geq 0$, as $n \rightarrow \infty$,

$$
\mathrm{P}\{n<\tau(u)<\infty\} \sim c H(u) \gamma^{n} n^{-3 / 2},
$$

where $c$ is a known constant and $H$ a function depending solely on $u$.

The quantity $c=\gamma c_{1} /(1-\gamma)$, with $c_{1}^{2}=\gamma /\left(2 \pi \omega^{2} \hat{K}^{\prime \prime}(-\omega)\right)$, is given explicitly. The function $H(u)$ can only be given in (complicated) implicit form, involving the quantities $\gamma$ and $\omega$. Hence, it is also affected by the interdependence between the claim sizes and the interarrival times. In Section 6 an explicit expression for $H(u), u \rightarrow \infty$, is derived, giving an intuition for this effect.

Let us have a closer look at the conditions of the theorem. Condition (i) is of course the balance condition, necessary for the eventual survival of the portfolio. The second condition has already been discussed in Section 3. Clearly, the existence of an adjustment coefficient $R$ is sufficient for both (ii) and (iii) to hold.

Example 5.1. (Example 3.1 continued: the independent case.) Assume that $\sigma_{K_{I}}=\sigma_{U}>0$. Then $-\omega$ is the solution to $\psi_{U}(\theta)=c \psi_{T}(-c \theta)$, where $\psi_{U}(\theta):=-\hat{F}_{U}^{\prime}(\theta) / \hat{F}_{U}(\theta)$ denotes the logarithmic derivative of $\hat{F}_{U}(\theta)$ (and $\psi_{T}(\theta)$ is defined analogously). Since $U$ and $T$ are nonnegative random variables, $\psi_{U}(\theta)$ is monotonically decreasing in $\theta \in\left(-\sigma_{U}, \infty\right)$ and $\psi_{T}(-c \theta)$ is monotonically increasing in $\theta \in(-\infty, 0]$. Since, at the origin, $\psi_{U}(0)=\mathrm{E}\{U\}<c$ and $c \psi_{T}(0)=\mathrm{E}\{T\}$, the existence and uniqueness of $-\omega$ are guaranteed. 


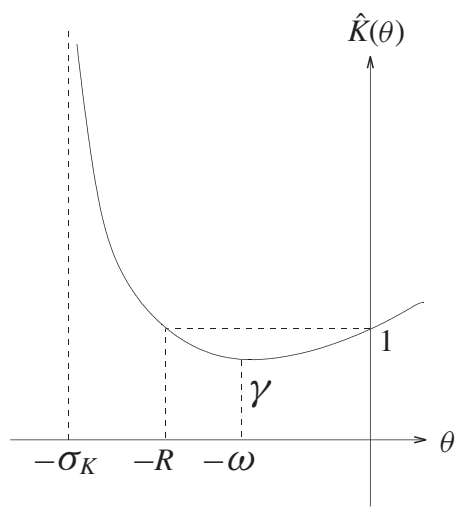

FIGURE 1: The Laplace transform $\hat{K}(\theta)$.

Let us now fix the marginals $U$ and $T$ again and consider the behavior of the crucial quantities $\gamma$ and $\omega$ in the presence of dependence. From (3.3) it follows that $\gamma<\gamma_{\text {I }}$ and $\gamma>\gamma_{\text {I }}$ for positively and, respectively, negatively quadrant-dependent $U$ and $T$, where $\gamma_{\text {I }}$ corresponds to the case of independence (and Remark 4.2 on other dependence concepts applies here accordingly). More generally, if $C_{1} \succ C_{2}$ (in terms of a concordance ordering), then $\gamma_{1} \leq \gamma_{2}$. However, similar inequalities cannot be established for $\omega$ and $\omega_{\mathrm{I}}$ (see, e.g. Example 5.7, below, where $\omega$ is insensitive to the degree of dependence). Since $\hat{K}^{\prime}(\theta)$ is analytic at $-\omega_{\mathrm{I}}$, if $\hat{K}^{\prime \prime}\left(-\omega_{\mathrm{I}}\right) \neq 0$ we thus obtain

$$
\begin{aligned}
-\omega & =-\omega_{\mathrm{I}}+\left.\sum_{n=1}^{\infty} \frac{\mathrm{d}^{n-1}}{\mathrm{~d} w^{n-1}}\left(\frac{w+\omega_{\mathrm{I}}}{\hat{K}^{\prime}(w)-\hat{K}^{\prime}\left(-\omega_{\mathrm{I}}\right)}\right)^{n}\right|_{w=-\omega_{\mathrm{I}}} \frac{\left(-\hat{K}^{\prime}\left(-\omega_{\mathrm{I}}\right)\right)^{n}}{n !} \\
& =-\omega_{\mathrm{I}}-\frac{\hat{K}^{\prime}\left(-\omega_{\mathrm{I}}\right)}{\hat{K}^{\prime \prime}\left(-\omega_{\mathrm{I}}\right)}+\frac{1}{2} \frac{\hat{K}^{\prime \prime \prime}\left(-\omega_{\mathrm{I}}\right) \hat{K}^{\prime}\left(-\omega_{\mathrm{I}}\right)^{2}}{\hat{K}^{\prime \prime}\left(-\omega_{\mathrm{I}}\right)^{3}}+\cdots,
\end{aligned}
$$

by Lagrange expansion. This series converges if $\omega-\omega_{\text {I }}$ is sufficiently small. By means of Bürmann's theorem (see [15, p. 128]), we can obtain information on the value of $\gamma$ directly in terms of properties of $\hat{K}$ at $-\omega_{\text {I }}$. In fact,

$$
\gamma=\hat{K}\left(-\omega_{\mathrm{I}}\right)+\left.\sum_{n=1}^{m-1} \frac{\mathrm{d}^{n-1}}{\mathrm{~d} w^{n-1}}\left(\hat{K}^{\prime}(w) \frac{w+\omega_{\mathrm{I}}}{\hat{K}^{\prime}(w)-\hat{K}^{\prime}\left(-\omega_{\mathrm{I}}\right)}\right)^{n}\right|_{w=-\omega_{\mathrm{I}}} \frac{\left(-\hat{K}^{\prime}\left(-\omega_{\mathrm{I}}\right)\right)^{n}}{n !}+R_{m},
$$

where the remainder term is given by

$$
R_{m}=\frac{1}{2 \pi \mathrm{i}} \int_{-\omega_{\mathrm{I}}}^{-\omega} \int_{D}\left(\frac{\hat{K}^{\prime}(-\omega)-\hat{K}^{\prime}\left(-\omega_{\mathrm{I}}\right)}{\hat{K}^{\prime}(t)-\hat{K}^{\prime}\left(-\omega_{\mathrm{I}}\right)}\right)^{m-1} \frac{\hat{K}^{\prime}(t) \hat{K}^{\prime \prime}(-\omega) \mathrm{d} t \mathrm{~d} \omega}{\hat{K}^{\prime}(t)-\hat{K}^{\prime}(-\omega)}
$$

and $D$ is a contour in the $t$-plane, enclosing the points $-\omega_{\mathrm{I}}$ and $-\omega$, such that the equation $\hat{K}^{\prime}(t)=\hat{K}^{\prime}(\zeta)$ has no roots inside or on $D$ except at $t=\zeta$, where $\zeta$ is any point inside $D$. The first few terms of (5.2) are thus given by

$$
\gamma=\hat{K}\left(-\omega_{\mathrm{I}}\right)-\frac{1}{2} \frac{\hat{K}^{\prime}\left(-\omega_{\mathrm{I}}\right)^{2}}{\hat{K}^{\prime \prime}\left(-\omega_{\mathrm{I}}\right)}-\frac{\hat{K}^{\prime}\left(-\omega_{\mathrm{I}}\right)^{3} \hat{K}^{\prime \prime \prime}\left(-\omega_{\mathrm{I}}\right)}{2 \hat{K}^{\prime \prime}\left(-\omega_{\mathrm{I}}\right)^{3}}+\cdots
$$


The above expansions provide an approach to obtaining sensitivity results on the degree of dependence of the quantities determining the asymptotic behavior of the risk process, if the Laplace transform $\hat{K}(\theta)$ is given for the dependent case. In some cases it might be possible to obtain an empirical Laplace transform from data sets of $U$ and $T$. In what follows we will illustrate the above result in several examples.

\subsection{Some general cases}

In quite a number of cases, a copula can be decomposed into a convex combination of two more fundamental copulas. Suppose that, for some quantity $\alpha \in(0,1)$, the distribution $K$ has copula, $C$, given by

$$
C(a, b)=\alpha C_{1}(a, b)+(1-\alpha) C_{2}(a, b),
$$

where, for $i=1,2, C_{i}$ is a copula linked to the distribution $K_{i}$ through (3.2). If $\sigma_{i}$ refers to the abscissa for $K_{i}, i=1,2$, then the corresponding abscissa for $K$ is given by $\sigma=\min \left(\sigma_{1}, \sigma_{2}\right)$. Moreover, on the interval $(-\sigma, 0]$ all three functions, $\hat{K}(\theta), \hat{K}_{1}(\theta)$, and $\hat{K}_{2}(\theta)$, are positive and convex. In particular, if, for $i=1,2, \hat{K}_{i}(\theta)$ has a minimum, $\gamma_{i}$, at $-\omega_{i}$, then the minimum, $\gamma$, of $\hat{K}(\theta)$ is attained at a value, $-\omega$, satisfying $\min \left(\omega_{1}, \omega_{2}\right) \leq \omega \leq \max \left(\omega_{1}, \omega_{2}\right)$. Moreover, $\gamma \geq \alpha \gamma_{1}+(1-\alpha) \gamma_{2}$.

Example 5.2. (The positive linear Spearman copula.) The positive linear Spearman copula has a particularly simple structure given by

$$
C_{\rho_{\mathrm{S}}}(a, b)= \begin{cases}\left(a+\rho_{\mathrm{S}}(1-a)\right) b, & b \leq a, \\ \left(b+\rho_{\mathrm{S}}(1-b)\right) a, & b>a,\end{cases}
$$

where we assume that $\rho_{\mathrm{S}} \geq 0$ (see, e.g. [7]). The name stems from the fact that the dependence parameter, $\rho_{\mathrm{S}}$, coincides with Spearman's rank correlation coefficient, which is a measure of concordance. Note that there is also a simple relation to Kendall's $\tau$, namely

$$
\tau=\frac{1}{3} \rho_{\mathrm{S}}\left(2+\operatorname{sgn}\left(\rho_{\mathrm{S}}\right) \rho_{\mathrm{S}}\right) .
$$

The positive linear Spearman copula is a convex combination of the independence copula and the comonotone copula:

$$
C_{\rho_{\mathrm{S}}}(a, b)=\left(1-\rho_{\mathrm{S}}\right) C_{\mathrm{I}}(a, b)+\rho_{\mathrm{S}} C_{\mathrm{M}}(a, b) .
$$

This copula has upper tail dependence with corresponding tail dependence coefficient

$$
\lambda:=\lim _{\alpha \rightarrow 1^{-}} \frac{1}{1-\alpha}\left(1-2 \alpha+C_{\rho_{\mathrm{S}}}(\alpha, \alpha)\right)=\rho_{\mathrm{S}}
$$

(see, e.g. [8, p. 33]).

If the dependence structure of $U$ and $T$ is governed by this copula, we obtain

$$
\hat{K}(\theta)=\left(1-\rho_{\mathrm{S}}\right) \hat{K}_{\mathrm{I}}(\theta)+\rho_{\mathrm{S}} \hat{K}_{\mathrm{M}}(\theta)
$$

From (5.3) it can immediately be seen that, for $\rho_{\mathrm{S}}<1$, the marginal distribution $U$ has to be super-exponential in order to satisfy condition (ii) of Theorem 5.1. Moreover, $\sigma_{K}=\sigma_{U}$ and $\omega$ is the solution to

$$
\frac{\hat{K}_{\mathrm{M}}^{\prime}(-\omega)}{\hat{K}_{\mathrm{I}}^{\prime}(-\omega)}=-\frac{1-\rho_{\mathrm{S}}}{\rho_{\mathrm{S}}}<0 .
$$


From this it follows that

$$
\omega_{\rho_{\mathrm{S}}}>\omega_{\mathrm{I}} \text { and } \gamma_{\rho_{\mathrm{S}}}<\gamma_{\mathrm{I}} .
$$

If, in addition, we assume there to be exponential marginal distributions $F_{U}(u)=1-\mathrm{e}^{-\lambda_{1} u}$ and $F_{T}(t)=1-\mathrm{e}^{-\lambda_{2} t}$, then, in order to satisfy condition (i) of Theorem 5.1 we must have $c \lambda_{1}>\lambda_{2}$. From (5.3), we obtain

$$
\hat{K}(\theta)=\left(1-\rho_{\mathrm{S}}\right) \frac{\lambda_{1}}{\lambda_{1}+\theta} \frac{\lambda_{2}}{\lambda_{2}-c \theta}+\frac{\lambda_{1} \rho_{\mathrm{S}}}{\theta\left(1-c \lambda_{1} / \lambda_{2}\right)+\lambda_{1}} .
$$

Example 5.3. (The negative linear Spearman copula.) We now assume that $\rho_{\mathrm{S}} \leq 0$. The copula is defined by

$$
C_{\rho_{\mathrm{S}}}(a, b)= \begin{cases}\left(1+\rho_{\mathrm{S}}\right) a b, & a+b \leq 1, \\ a b+\rho_{\mathrm{S}}(1-a)(1-b), & a+b>1\end{cases}
$$

The simple relation to Kendall's $\tau$ again holds, and the negative linear Spearman copula is again a convex combination, this time of $C_{\mathrm{I}}(a, b)$ and $C_{\mathrm{W}}(a, b)$ :

$$
C_{\rho_{\mathrm{S}}}(a, b)=\left(1+\rho_{\mathrm{S}}\right) C_{\mathrm{I}}(a, b)-\rho_{\mathrm{S}} C_{\mathrm{W}}(a, b) .
$$

Accordingly, we have

$$
\hat{K}(\theta)=\left(1+\rho_{\mathrm{S}}\right) \hat{K}_{\mathrm{I}}(\theta)-\rho_{\mathrm{S}} \hat{K}_{\mathrm{W}}(\theta) .
$$

Thus, for $-1<\rho_{\mathrm{S}}<0$, the marginal distribution $U$ has to be super-exponential in order to satisfy condition (ii) of Theorem 5.1. Moreover, $\sigma_{K} \leq \sigma_{U}$ and $\omega$ is the solution to

$$
\frac{\hat{K}_{\mathrm{W}}^{\prime}(-\omega)}{\hat{K}_{\mathrm{I}}^{\prime}(-\omega)}=\frac{1+\rho_{\mathrm{S}}}{\rho_{\mathrm{S}}}<0
$$

whence

$$
\omega_{\rho_{\mathrm{S}}}<\omega_{\mathrm{I}} \text { and } \gamma_{\rho_{\mathrm{S}}}>\gamma_{\mathrm{I}}
$$

For exponential marginals, we obtain

$$
\hat{K}(\theta)=\left(1-\rho_{\mathrm{S}}\right) \frac{\lambda_{1}}{\lambda_{1}+\theta} \frac{\lambda_{2}}{\lambda_{2}-c \theta}-\rho_{\mathrm{S}} B\left(1+\frac{\theta}{\lambda_{1}}, 1-\frac{c \theta}{\lambda_{2}}\right),
$$

in terms of a beta function.

Example 5.4. (The Farlie-Gumbel-Morgenstern copula.) This is an analytically simple and at the same time absolutely continuous copula given by

$$
C(a, b)=a b\left(1+3 \rho_{\mathrm{S}}(1-a)(1-b)\right),
$$

where $\rho_{\mathrm{S}},-\frac{1}{3} \leq \rho_{\mathrm{S}} \leq \frac{1}{3}$, is again Spearman's rank correlation coefficient (for Kendall's $\tau$ we have $\tau=2 \rho_{\mathrm{S}} / 3$ ). Thus, this copula allows for weak dependence only. For exponential marginals with parameters as above we obtain

$$
\hat{K}(\theta)=\frac{\lambda_{1} \lambda_{2}\left(\left(\theta+2 \lambda_{1}\right)\left(2 \lambda_{2}-c \theta\right)-3 c \rho_{\mathrm{S}} \theta^{2}\right)}{\left(\theta+\lambda_{1}\right)\left(\theta+2 \lambda_{1}\right)\left(\lambda_{2}-c \theta\right)\left(2 \lambda_{2}-c \theta\right)},
$$

and the determination of $R$ and $\omega$ leads to polynomial equations of orders four and five, respectively. 
Example 5.5. (Archimedean copulas.) Bivariate Archimedean copulas are an important subclass of copulas defined by

$$
C(a, b)=\phi^{[-1]}(\phi(a)+\phi(b)), \quad 0 \leq a, b \leq 1,
$$

where the generator $\phi(t)$ is a continuous, convex, strictly decreasing function from $[0,1]$ to $[0, \infty]$ such that $\phi(1)=0$, and $\phi^{[-1]}$ denotes the pseudo-inverse of $\phi$, defined by

$$
\phi^{[-1]}(t)= \begin{cases}\phi^{-1}(t), & 0 \leq t \leq \phi(0) \\ 0, & \phi(0) \leq t \leq \infty\end{cases}
$$

The concordance measure $\tau$ can easily be determined from the generator through

$$
\tau=1-4 \int_{0}^{\infty} s\left(\frac{\mathrm{d}}{\mathrm{d} s} \phi^{[-1]}(s)\right)^{2} \mathrm{~d} s .
$$

Techniques for fitting these types of copula to given bivariate data sets can be found in [6]. Here we will just state a general monotonicity result. Let us again assume that the marginal distributions of $U$ and $T$ are fixed. Since an Archimedean copula, $C_{1}$, dominates another Archimedean copula, $C_{2}$, in concordance order if and only if the function $\phi_{2} \circ \phi_{1}^{[-1]}$ is subadditive (see [10, p. 109]), representation (3.2) implies that $R_{1}>R_{2}$ and $\gamma_{1}<\gamma_{2}$ whenever the above subadditivity holds. Sufficient conditions for the latter are for instance the concavity of $\phi_{2} \circ \phi_{1}^{[-1]}$ or the nondecrease on $(0,1)$ either of $\phi_{1} / \phi_{2}$ or (given that $\phi_{1}$ and $\phi_{2}$ are continuously differentiable on $(0,1))$ of $\phi_{1}^{\prime} / \phi_{2}^{\prime}$. In particular, the above monotonicity results apply to ordered families of copulas (see [10, Section 4.4]).

\subsection{Specific cases}

We now deal with a few parametric bivariate distributions for which $\omega$ and $\gamma$ can be evaluated explicitly as functions of the dependence parameter.

Example 5.6. (Moran and Downton's bivariate exponential.) The joint density function is given by

$$
f_{U, T}(u, t)=\frac{\lambda_{1} \lambda_{2}}{1-\rho} I_{0}\left(\frac{2 \sqrt{\rho \lambda_{1} \lambda_{2} u t}}{1-\rho}\right) \exp \left(-\frac{\lambda_{1} u+\lambda_{2} t}{1-\rho}\right),
$$

where $I_{0}(z)=\sum_{j=0}^{\infty}\left(1 / j !^{2}\right)(z / 2)^{2 j}$ is the modified Bessel function of the first kind and order zero; $\rho, 0 \leq \rho \leq 1$, is Pearson's correlation coefficient; and $\lambda_{1}, \lambda_{2}, u, t>0$ (see [9]). Here the marginal distributions $U$ and $T$ are exponential with respective parameters $\lambda_{1}$ and $\lambda_{2}$. From the particularly simple structure of the joint moment generating function we obtain

$$
\hat{K}(\theta)=\frac{\lambda_{1} \lambda_{2}}{c \theta^{2}(\rho-1)+\theta\left(\lambda_{2}-c \lambda_{1}\right)+\lambda_{1} \lambda_{2}},
$$

and thus have

$$
\sigma_{K}=\frac{\lambda_{2}-c \lambda_{1}-\sqrt{\left(c \lambda_{1}-\lambda_{2}\right)^{2}+4 c \lambda_{1} \lambda_{2}(1-\rho)}}{2 c(1-\rho)} .
$$

The adjustment coefficient is now given by

$$
R=\frac{c \lambda_{1}-\lambda_{2}}{c(1-\rho)}
$$


which is positive if $c \lambda_{1}>\lambda_{2}$. However, the latter is just the net balance condition (i) for the marginal distributions. From (5.4) it follows that

$$
\omega=\frac{\lambda_{2}-c \lambda_{1}}{2 c(\rho-1)}=\frac{R}{2} .
$$

Furthermore, we have

$$
\gamma=\hat{K}(-\omega)=\frac{\lambda_{1} \lambda_{2}}{\lambda_{1} \lambda_{2}+\left(c \lambda_{1}-\lambda_{2}\right)^{2} / 4 c(1-\rho)} .
$$

Example 5.7. (Kibble and Moran's bivariate gamma.) This symmetric bivariate distribution with standard gamma marginals (shape parameter $\alpha>0$ ) is defined through its joint moment generating function,

$$
\mathrm{E}\left\{\mathrm{e}^{t_{1} U+t_{2} T}\right\}=\left(1-\frac{\beta+1}{\beta} t_{1}-\frac{\beta+1}{\beta} t_{2}+\frac{\beta+1}{\beta} t_{1} t_{2}\right)^{-\alpha} .
$$

Here $\beta>0$ is the dependence parameter, and Pearson's correlation coefficient is given by $1 /(1+\beta)$. We thus have

$$
\hat{K}(\theta)=\left(1-\frac{\beta+1}{\beta}\left((c-1) \theta+c \theta^{2}\right)\right)^{-\alpha}
$$

and

$$
\sigma_{K}=\frac{(1-c)(1+\beta)-\sqrt{(1-c)^{2}(1+\beta)^{2}+4 c \beta(1+\beta)}}{2 c(1+\beta)} .
$$

It follows easily that $R=(c-1) / c$ and

$$
\omega=\frac{c-1}{2 c}=\frac{R}{2}
$$

which is positive, since condition (i) amounts to $c>1$ in this case. Note that $R$ and $\omega$ are independent of the dependence parameter $\beta$. The crucial quantity $\gamma$ depends on $\beta$ and is given by

$$
\gamma=\left(\frac{4 c \beta}{1+2 c(\beta-1)+\beta+c^{2}(1+\beta)}\right)^{\alpha} .
$$

Example 5.8. (Marshall and Olkin's bivariate exponential.) The distribution is defined by

$$
\mathrm{P}\{U>u, T>t\}=\exp \left(-\lambda_{1} u-\lambda_{2} t-\lambda_{3} \max (u, t)\right), \quad u, t>0 .
$$

In this example, the exponential marginal distributions, with parameters $\lambda_{1}+\lambda_{3}$ and $\lambda_{2}+\lambda_{3}$, are functions of the degree of dependence. Pearson's correlation coefficient is $\lambda_{3} /\left(\lambda_{1}+\lambda_{2}+\lambda_{3}\right)$ (see [9]). We find that

$$
\hat{K}(\theta)=\frac{\left(\lambda_{1}+\lambda_{2}+\lambda_{3}+\theta(c-1)\right)\left(\lambda_{1}+\lambda_{3}\right)\left(\lambda_{2}+\lambda_{3}\right)-c \lambda_{3} \theta^{2}}{\left(\lambda_{1}+\lambda_{3}+\theta\right)\left(\lambda_{2}+\lambda_{3}-c \theta\right)\left(\lambda_{1}+\lambda_{2}+\lambda_{3}+\theta(1-c)\right)} .
$$

In this case both $R$ and $\omega$ are solutions to polynomial equations of third order. 
Other bivariate distributions that lead to polynomial equations of low order for $\omega$ include Freund's bivariate exponential distribution (order three) and the bivariate gamma of Cheriyan and Ramabhadran (order two) (see [9] for their definitions).

Example 5.9. (The bivariate normal distribution.) Although the Gaussian case with underlying Gaussian copula and joint density

$$
\begin{aligned}
f_{U, T}(u, t)= & \frac{1}{2 \pi \sigma_{1} \sigma_{2} \sqrt{1-\rho^{2}}} \\
& \times \exp \left(-\left\{\left(\frac{u-\mu_{1}}{\sigma_{1}}\right)^{2}-2 \rho \frac{u-\mu_{1}}{\sigma_{1}} \frac{t-\mu_{2}}{\sigma_{2}}+\left(\frac{t-\mu_{2}}{\sigma_{2}}\right)^{2}\right\} \frac{1}{2\left(1-\rho^{2}\right)}\right)
\end{aligned}
$$

(where $\rho \in[-1,1]$ denotes the correlation coefficient) may not be a suitable model, since the claim sizes and interarrival times can become negative, we include it here because it is often used as an approximation for a bivariate distribution with light tails. It is straightforward to see that, in this case,

$$
\hat{K}(\theta)=\exp \left(-\theta \mathrm{E}\{X\}+\frac{\theta^{2}}{2} \operatorname{var}(X)\right),
$$

where $\mathrm{E}\{X\}=\mu_{1}-c \mu_{2}$ and $\operatorname{var}(X)=\sigma_{1}^{2}-2 \rho \sigma_{1} \sigma_{2} c+\sigma_{2}^{2} c^{2}$. Hence, we obtain the explicit expressions

$$
R=\frac{2 \mathrm{E}\{X\}}{\operatorname{var}(X)}, \quad \omega=\frac{R}{2}, \quad \text { and } \quad \gamma=\mathrm{e}^{-\mathrm{E}^{2}\{X\} / \operatorname{var}(X)} .
$$

\subsection{Conditioning on the event of ruin}

If we condition on the occurrence of ruin then, if the adjustment coefficient $R$ exists, it is well known that the asymptotic behavior of the random walk $S_{n}$ can be studied in terms of its associated random walk $\tilde{S}_{n}$, defined by (4.1). For large $u$, we have

$$
\mathrm{P}\left\{X_{1} \leq x_{1}, \ldots, X_{n} \leq x_{n} \mid \tau(u)<\infty\right\} \sim \mathrm{P}\left\{\tilde{X}_{1} \leq x_{1}, \ldots, \tilde{X}_{n} \leq x_{n}\right\}
$$

(see Asmussen [1]), meaning that the properties of the surplus process conditioned on ruin are determined by the Laplace transform $\hat{K}(\theta)$ shifted by $-R$ to the right. For instance, $\mathrm{E}\left\{\tilde{X}_{i}\right\}=-\hat{K}^{\prime}(-R)>0$. Thus, conditioned on the occurrence of ruin in finite time, the random walk has a positive drift. By adapting Theorem B of [14] to our situation, for large $u$ we thus obtain

$$
\mathrm{P}\{\tau(u)>n \mid \tau(u)<\infty\} \sim H_{2}(u) \gamma^{n} n^{-3 / 2} \text { as } n \rightarrow \infty .
$$

Here $\mathrm{H}_{2}(u)$ is a function, depending only on $u$, that can be expressed in terms of quantities related to the random walk (see [14]). Hence, by studying the behavior of $\gamma$ for dependent marginals $U$ and $T$, as in the previous sections, we can also derive rather sharp asymptotic results on the finite-time ruin probability conditioned on the event of ruin.

\section{The function $H$}

This section is devoted to a closer look at the function $H$, whose existence we used in (5.1), but whose properties have not been revealed. First, it follows from [13] that $H(0)=\mathrm{e}^{-B(1 / \gamma)}$. For $u>0$, the function $H$ was given in a rather complicated form in [13]. However, if we use the Markovian structure of the random walk, then we are able to give a much neater interpretation of $H$ in terms of its Laplace transform. 
Let us find the first time at which the random walk hits its positive maximum. Introduce the auxiliary quantities

$$
u_{n}(x):=\mathrm{P}\left\{S_{1}>0, S_{2}>0, \ldots, S_{n-1}>0,0<S_{n} \leq x\right\}
$$

and $u(x):=\sum_{n=1}^{\infty} u_{n}(x)$. If we define

$$
L_{0}=0, \quad L_{n}=\min \left\{r \geq 0: S_{r}=\max _{0 \leq k \leq n} S_{k}\right\}
$$

then it is clear that

$$
u_{n}(x):=\mathrm{P}\left\{L_{n}=n, S_{n} \leq x\right\} .
$$

If we link these portfolio variables with the random walk, then the Markovian character of the latter allows us to write

$$
\mathrm{P}\{n<\tau(u)<\infty\}=\sum_{k=0}^{n} \mathrm{P}\{n-k<\tau(0)<\infty\} \mathrm{P}\left\{L_{k}=k, S_{k} \leq u\right\}
$$

(see, e.g. [5, p. 573]). Therefore,

$$
\frac{\mathrm{P}\{n<\tau(u)<\infty\}}{\mathrm{P}\{n<\tau(0)<\infty\}}=\sum_{k=0}^{n} \frac{\mathrm{P}\{n-k<\tau(0)<\infty\}}{\mathrm{P}\{n<\tau(0)<\infty\}} u_{k}(u) .
$$

However, from (5.1), for $u=0$ we immediately see that

$$
\lim _{n \uparrow \infty} \frac{\mathrm{P}\{n-k<\tau(0)<\infty\}}{\mathrm{P}\{(n<\tau(0)<\infty\}}=\gamma^{-k}
$$

for each fixed $k$, and it then follows that

$$
\frac{H(u)}{H(0)}=\lim _{n \uparrow \infty} \frac{\mathrm{P}\{n<\tau(u)<\infty\}}{\mathrm{P}\{n<\tau(0)<\infty\}}=\sum_{k=0}^{\infty} \gamma^{-k} u_{k}(u) .
$$

A fundamental relation is the following Spitzer-Baxter identity, which gives the hybrid transform of the sequence $\left\{u_{n}(x)\right\}$. Let

$$
U(s, x):=\sum_{n=0}^{\infty} u_{n}(x) s^{n} .
$$

Then, for $|s|<1$ and $\zeta \in \mathbb{R}$,

$$
\tilde{u}(s, \zeta):=\int_{0}^{\infty} \mathrm{e}^{\mathrm{i} \zeta x} U(s, \mathrm{~d} x)=\exp \left(\sum_{n=1}^{\infty} \frac{s^{n}}{n} \int_{0+}^{\infty} \mathrm{e}^{\mathrm{i} \zeta x} \mathrm{~d} K^{* n}(x)\right) .
$$

In view of (2.1) we thus have the following remarkable formula for the Laplace transform of $H$, which resembles (2.4) closely:

$$
\hat{H}(\theta)=\frac{\mathrm{e}^{-B(1 / \gamma)}}{1-\mathrm{E}\left\{\gamma^{-N} \mathrm{e}^{-\theta S_{N}}\right\}} .
$$


To get a better look at the behavior of the function $H$ we introduce associated random walks. For any $\delta \in\left(-\sigma_{K}, 0\right]$, define the distribution

$$
K_{\delta}(x)=\frac{1}{\hat{K}(\delta)} \int_{-\infty}^{x} \mathrm{e}^{-\delta u} \mathrm{~d} K(u)
$$

Its bilateral Laplace transform is given by

$$
\hat{K}_{\delta}(\theta)=\frac{\hat{K}(\theta+\delta)}{\hat{K}(\delta)} .
$$

Recall the Laplace transform analogue of expression (2.2) for the random walk generated by $K_{\delta}:$

$$
1-s \hat{K}_{\delta}(\theta)=\left(1-\chi_{\delta}(s, \mathrm{i} \theta)\right)\left(1-\tilde{\chi}_{\delta}(s, \mathrm{i} \theta)\right) .
$$

However, $\hat{K}(\theta)$ also has its own decomposition. Hence,

$$
1-\frac{s}{\hat{K}(\delta)} \hat{K}(\theta+\delta)=\left(1-\chi\left(\frac{s}{\hat{K}(\delta)}, \mathrm{i}(\theta+\delta)\right)\right)\left(1-\tilde{\chi}\left(\frac{s}{\hat{K}(\delta)}, \mathrm{i}(\theta+\delta)\right)\right) .
$$

By the uniqueness of the Wiener-Hopf decomposition, it follows that

$$
\chi_{\delta}(s, \mathrm{i} \theta)=\chi\left(\frac{s}{\hat{K}(\delta)}, \mathrm{i}(\theta+\delta)\right)
$$

or, in terms of ladder quantities,

$$
\mathrm{E}\left\{s^{N_{\delta}} \mathrm{e}^{\left.-\theta S_{N_{\delta}}\right\}}=\mathrm{E}\left\{\left(\frac{s}{\hat{K}(\delta)}\right)^{N} \mathrm{e}^{-(\theta+\delta) S_{N}}\right\}\right.
$$

where $N_{\delta}$ is the first upgoing ladder index for the associated random walk generated by the distribution $K_{\delta}$, and $S_{N_{\delta}}$ is the corresponding ladder height.

If we now compare this formula with (6.1), the substitution $\theta=\delta+\beta$ leads to the equality

$$
\int_{0}^{\infty} \mathrm{e}^{-\beta x} \mathrm{~d}\left(\int_{0}^{x} \mathrm{e}^{-\delta u} \mathrm{~d} H(u)\right)=\frac{\mathrm{e}^{-B(1 / \gamma)}}{1-\mathrm{E}\left\{(\hat{K}(\delta) / \gamma)^{N_{\delta}} \mathrm{e}^{-\beta S_{N_{\delta}}}\right\}},
$$

which is valid for $-\sigma_{K}-\delta<\beta<-\delta$. In view of (2.4) it then looks natural to choose $\delta$ in such a way that $\hat{K}(\delta)=\gamma$, or $\delta=-\omega$, since then

$$
\int_{0}^{\infty} \mathrm{e}^{-\beta x} \mathrm{~d}\left(\int_{0}^{x} \mathrm{e}^{\omega u} \mathrm{~d} H(u)\right)=\frac{\mathrm{e}^{-B(1 / \gamma)}}{1-\mathrm{E}\left\{\mathrm{e}^{-\beta S_{N_{\omega}}}\right\}},
$$

which is valid for $-\sigma_{K}+\omega<\beta<\omega$.

However, we can now repeat the procedure from Section 4. Using a similar application of the renewal theorem leads to the following asymptotic expression:

$$
1-H(u) \sim \frac{\mathrm{e}^{-B(1 / \gamma)}}{\omega \mathrm{E}\left\{S_{N_{\omega}}\right\}} \mathrm{e}^{-\omega u} \quad \text { as } u \rightarrow \infty .
$$




\section{Conclusion}

The random walk approach presented in this paper has allowed us to extend several rather explicit asymptotic results for the independent risk process to a dependent framework. Moreover, the introduction of copula functions has enabled us to study the dependence structures separately from the marginal behavior of the distributions involved. However, the present paper is just an attempt to obtain a clearer picture of the impact of dependence in risk theory, and many questions remain open to further study. For instance, one might attempt a similar study for heavy-tailed claims, possibly based on results of Baltrūnas [3].

\section{Acknowledgements}

We are grateful for the comments of the referees that helped to improve the presentation of the paper. This research was carried out while the first author was visiting the University Center for Statistics of the Katholieke Universiteit Leuven through Fellowship F/03/035. He would like to thank the center for its hospitality.

\section{References}

[1] Asmussen, S. (1982). Conditioned limit theorems relating a random walk to its associate, with applications to risk reserve processes and the GI/G/1 queue. Adv. Appl. Prob. 14, 143-170.

[2] Asmussen, S. (2000). Ruin Probabilities. World Scientific, Singapore.

[3] BaltrūNas, A. (2001). Some asymptotic results for transient random walks with applications to insurance risk. J. Appl. Prob. 38, 108-121.

[4] Breiman, L. (1968). Probability. Addison-Wesley, Reading, MA.

[5] Feller, W. (1966). An Introduction to Probability Theory and its Applications, Vol. 2. John Wiley, New York.

[6] Genest, C. And Rivest, L. (1993). Statistical inference procedures for bivariate Archimedean copulas. J. Amer. Statist. Assoc. 88, 1034-1043.

[7] Hürlimann, W. (2000). A Spearman multivariate distribution with fixed margins - theory and applications. Preprint.

[8] Joe, H. (1997). Multivariate Models and Dependence Concepts. Chapman and Hall, London.

[9] Kotz, S., Balakrishnan, N. And Johnson, N. L. (2000). Continuous Multivariate Distributions, Vol. 1, Models and Applications. John Wiley, New York.

[10] Nelsen, R. (1999). An Introduction to Copulas. Springer, Berlin.

[11] Prabhu, N. U. (1965). Stochastic Processes. Basic Theory and its Applications. Macmillan, New York.

[12] Rolski, T., Schmidli, H., Schmidt, V. And Teugels, J. L. (1999). Stochastic Processes for Insurance and Finance. John Wiley, Chichester.

[13] Veraverbeke, N. and Teugels, J. L. (1975). The exponential rate of convergence of the distribution of the maximum of a random walk. J. Appl. Prob. 12, 279-288.

[14] Veraverbeke, N. and Teugels, J. L. (1976). The exponential rate of convergence of the distribution of the maximum of a random walk. II. J. Appl. Prob. 13, 733-740.

[15] Whittaker, E. And Watson, G. (1963). A Course of Modern Analysis, 4th edn. Cambridge University Press.

[16] Widder, D. (1942). The Laplace Transform. Princeton University Press. 